\title{
New visible spectra and mineralogical assessment of (21) Lutetia, a target of the Rosetta mission ${ }^{\star}$
}

\author{
M. Lazzarin ${ }^{1}$, S. Marchi ${ }^{1}$, L. V. Moroz ${ }^{2,3}$, and S. Magrin ${ }^{1}$ \\ 1 Dipartimento di Astronomia, Università di Padova, Vicolo dell'Osservatorio 3, 35122 Padova, Italy \\ e-mail: [monica.lazzarin; simone.marchi; sara.magrin.1]@unipd.it \\ 2 Institute of Planetology, University of Münster, Wilhelm-Klemm Str. 10, 48149 Münster, Germany \\ 3 German Aerospace Center (DLR), Institute of Planetary Research, Rutherfordstr. 2, 12489 Berlin, Germany \\ e-mail: Ljuba.Moroz@dlr.de
}

Received 13 May 2008 / Accepted 4 December 2008

ABSTRACT

\begin{abstract}
The Rosetta spacecraft, launched on March 2nd 2004, in the course of its journey to the comet 67P/Churyumov-Gerasimenko (encounter foreseen in 2014), will fly past two asteroids: (2867) Steins and (21) Lutetia. On September 5th 2008 (2867) Steins was encountered. In this paper, we present two visible spectra of (21) Lutetia of different spectral resolutions covering the spectral ranges where possible absorption bands were previously revealed by Lazzarin and collaborators. We confirm detection of a broad complex feature between 0.45 and $0.55 \mu \mathrm{m}$ and two narrower features around 0.47 and $0.52 \mu \mathrm{m}$. We discuss possible assignments of these bands and suggest that they might originate from electronic transitions in pyroxenes, although unambiguous identification is difficult and the published thermal infrared (TIR) spectrum of (21) Lutetia suggests that pyroxene cannot be the dominant silicate component at its surface. Furthermore, we discuss the published spectra of (21) Lutetia in the range from near-UV to thermal infrared. We conclude that carbonaceous meteorites (chondrites and achondrites) appear to be the closest meteorite analogues of (21) Lutetia, based on the observed spectral features. Among these meteorites, metal-rich carbonaceous chondrites seem to be the most plausible analogue materials.
\end{abstract}

Key words. minor planets, asteroids - techniques: spectroscopic

\section{Introduction}

The International Rosetta Mission, successfully launched on March 2nd 2004, will encounter comet 67P/ChuryumovGerasimenko, the principal target of the mission, in 2014. The Rosetta mission was also designed to fly-by two asteroids during its long journey. (2867) Steins was encountered on 5th September 2008 at a minimum distance of about $800 \mathrm{~km}$, and (21) Lutetia will be encountered on July 10th, 2010 at about $3000 \mathrm{~km}$ at a speed of 15 kilometers per second. Rosetta will attempt to answer the main questions related to the origin and evolution of the Solar System. The constituents of the minor bodies, especially comets, contain the record of the chemical and physical primordial processes of the formation of our planetary system. In particular, the encounter of Rosetta with the asteroids will be important to obtaining detailed information about the dynamic properties, surface morphology, and composition of the two objects.

The targets of Rosetta have been extensively investigated by ground-based observational campaigns. The goal has been to obtain as much information as possible (rotation period, pole orientation, surface composition) to define the observational strategies of the spacecraft as accurately as possible.

On January, the 2nd and 3rd 2007 Rosetta imaged the asteroid (21) Lutetia from a distance of about 1.64 AU. The on-board camera OSIRIS imaged the asteroid passing through its field of view during the spacecraft's gradual approach to Mars.

^ Based on observations performed at ESO, program n. 71.C-0157, P.I. M. Lazzarin.
Steins and Lutetia are rather different objects. Steins is a small body: with an absolute magnitude of 13.18 (Hicks et al. 2004) and a polarimetric albedo of $0.45 \pm 0.10$, a diameter of approximately $4.6 \mathrm{~km}$ has been determined. The successful flyby of Steins (5 Sept. 2008) by Rosetta will provide, apart from other information, confirmation or not of the ground-based parameters that have so far been obtained. Lutetia is a larger asteroid with a diameter of $95.5 \pm 4.1 \mathrm{~km}$, as determined by IRAS (Tedesco et al. 1992), that has been found to have an albedo of $0.22 \pm 0.02$ and a synodic period of $8.17 \pm 0.01 \mathrm{~h}$ (Zappala et al. 1984; Dotto et al. 1992; Lagerkvist et al. 1995). Information about its shape, pole coordinates, and a prograde sense of rotation was reported by Magri et al. (1999). On the basis of available observations, Torppa et al. (2003) determined new pole coordinates and a model shape (sharp and irregular) with global dimensions $a / b=1.2$ and $b / c=1.2$. In spite of several types of investigation (polarimetric, visible and near-infrared spectroscopy) obtained until now, the taxonomic classification of Lutetia has not yet been completely defined. Zellner et al. (1985) classified Lutetia as an X-type, while analysis of the ECAS and IRAS thermal albedo data inferred an M-type (Barucci et al. 1987; Tholen \& Barucci 1989). Hiroi et al. (1993) found a good match between a Lutetia spectrum and that of the iron meteorite Mundrabilla, and the analysis of the SMASSII spectroscopic data favors classification of Lutetia as an Xk-type (Bus \& Binzel 2002). Lazzaro et al. (2004) also determined an X-classification for Lutetia. Howell et al. (1994) and Burbine \& Binzel (2002) observed Lutetia in the near-infrared and found that it has an unusual flat spectrum compared to other M-type asteroids. 
Birlan et al. (2004) and Barucci et al. (2005) found a good match between new visible and near-infrared spectra of Lutetia and spectra of carbonaceous chondrites, usually similar to C-type asteroids. NIR spectroscopy (Birlan et al. 2006) confirmed a similarity with CV-CO meteorites. Polarimetric observations supported the idea that the composition of Lutetia is similar to that of carbonaceous chondrite meteorites (Belskaya \& Lagerkvist 1996). Magri et al. (1999) inferred a low radar albedo for Lutetia that excluded the extensive exposure of bright pure metal on its surface. Mueller et al. (2006) and Shepard et al. (2005) published an albedo value consistent with similar measurements for M type asteroids and measurements inferred from IRAS data. However, in spite of the several measurements that have been completed, the precise value of the albedo still remains a matter of debate. On the basis of near IR observations in the $0.8-2.5 \mu \mathrm{m}$ interval Nedelcu et al. (2007) again inferred a composition consistent with that of a primitive body. A similarity with carbonaceous chondrites was found from Spitzer observations of the asteroid (Barucci et al. 2008). Rivkin et al. (2000) reported the detection of the $3 \mu \mathrm{m}$ absorption feature due to water of hydration, typical of hydrated carbonaceous meteorites.

Lazzarin et al. (2004b) acquired three visible spectra of Lutetia covering the entire rotational period of the object with the aim of discovering possible surface composition variations. The spectra obtained agree with a primitive composition and we found two main absorption features around 0.43 and $0.51 \mu \mathrm{m}$ that we tentatively attributed to ferric-iron spin forbidden absorptions present in minerals such as phyllosilicates, a product of the aqueous alteration process. Prokof'eva et al. (2005) detected absorption features between 0.44 and $0.67 \mu \mathrm{m}$ that they attributed to hydrated minerals. We present the analysis of two new visible spectra of Lutetia taken to confirm or exclude the presence of these absorption bands and possibly clarify their origin. In particular, we investigated the spectral region where these bands were detected by using a higher spectral resolution. A more precise knowledge of the surface composition of Lutetia is particularly important to the definition of the observational strategy of the Rosetta mission.

\section{Observations, data reduction and discussion}

We obtained two visible spectra of Lutetia in the range $0.45-0.70 \mu \mathrm{m}$ on December 7th 2004, with the ESO-NTT at La Silla, Chile (Fig. 1). The NTT was equipped with EMMI (ESO Multi-Mode Instrument), and was operated in the lowmedium resolution mode with the GRISM \#1 and the GRISM \#5 respectively and a slit width of $5^{\prime \prime}$ (corresponding to a resolving power of about 250 and 1000, respectively), chosen to minimize the differential refraction due to the atmosphere. The slit was oriented along the parallactic angle. Lutetia was also observed in the course of another observational program; a detailed description of the data reduction for these observations was given by Lazzarin et al. (2004a).

To minimize atmospheric extinction effects, we obtained spectra of different solar analogs at different airmasses during the night. The solar analogs spectra revealed negligible differences. The ratio between their spectra has a maximum deviation of less than $5 \%$ around 1 . The reflectivity was inferred by dividing the asteroid spectrum by the solar analog Landolt 93-101 and normalizing it at $0.55 \mu \mathrm{m}$.

As reported in Lazzarin et al. (2004b), we previously found absorption features in the spectra of Lutetia. We therefore attempted to investigate in detail the spectral region where the bands had previously been identified, by recording a spectrum

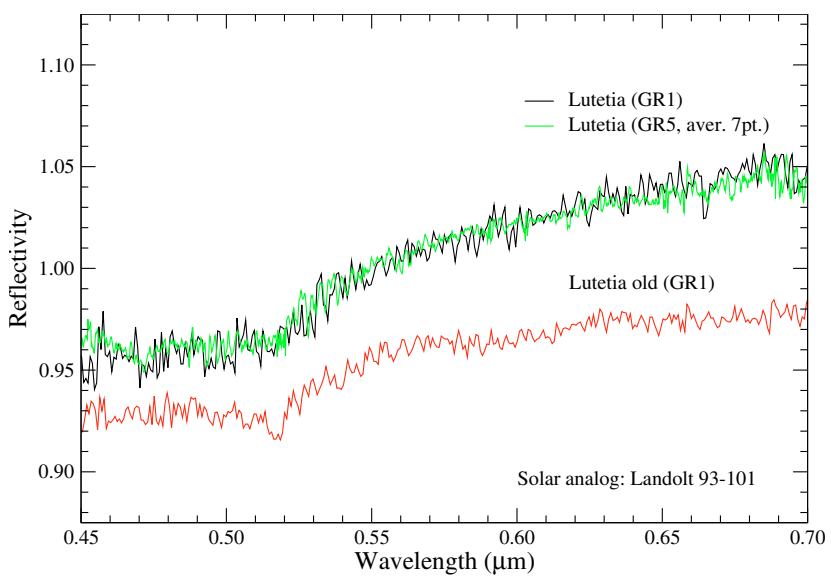

Fig. 1. Spectra of Lutetia taken with EMMI at NTT. Lower spectrum was observed in May 2003 with GRISM \#1 while upper spectra were observed in December 2004 with GRISM \#1 and GRISM \#5.

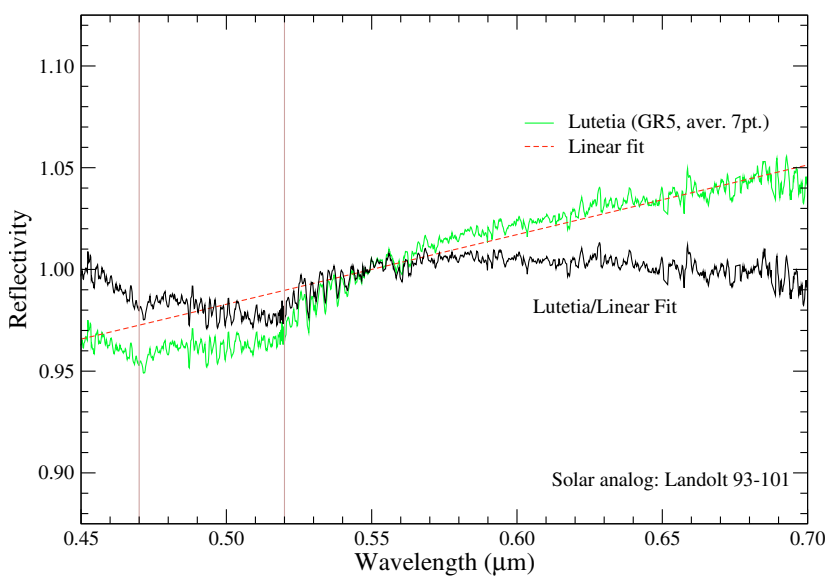

Fig. 2. Spectrum of Lutetia taken with the GRISM \#5 divided by a linear fit to emphasize the absorption bands. Vertical lines are placed at 0.47 and $0.52 \mu \mathrm{m}$.

of Lutetia with a higher spectral resolution between 0.45 and $0.70 \mu \mathrm{m}$. For comparison, we obtained during the same night a spectrum of Lutetia with low resolution, which appears similar to the higher resolution one, as shown in Fig. 1.

In our previous work (Lazzarin et al. 2004b), we identified two main absorption bands around 0.43 and $0.51 \mu \mathrm{m}$ that we tentatively attributed to a ferric iron spin-forbidden absorption present in hydrated minerals, such as phyllosilicates or hydrated ferric sulfate, (Vilas et al. 1993) and to crystalline iron oxides (Bell et al. 1989; Soderblom 1992) or to porphyrins, carbon-rich compounds indicative of a primitive composition (Luu \& Jewitt 1990), respectively. Prokof'eva et al. (2005) found a similar absorption band around $0.43-0.44 \mu \mathrm{m}$ that they attributed to phyllosilicates of the serpentine type.

The two new spectra confirm the presence of the absorption feature at $0.51 \mu \mathrm{m}$. However, we could not investigate the region shortward of $0.45 \mu \mathrm{m}$ because the solar analogues exhibited several differences at those wavelengths and we preferred to discard the short-wavelength range of the spectra. In this case, we cannot confirm the band around $0.43 \mu \mathrm{m}$ reported previously. However, we detected a narrow feature around $0.47 \mu \mathrm{m}$ and a feature around $0.52 \mu \mathrm{m}$ included in a wider depression between 0.45 and about $0.55 \mu \mathrm{m}$ (see Fig. 2). Since the observation of Lutetia was part of another observational program, we checked the spectra of other objects observed during the same 
night using the same solar analogues. These object spectra do not show evidence of these features, so even if they are subtle, we are confident that they are true features and not artifacts.

The broad inflection between 0.45 and $0.55 \mu \mathrm{m}$ may be explained by superposition of many absorption bands due to spinallowed and spin-forbidden crystal field transitions, and chargetransfer transitions. These inflections are observed in spectra of some pyroxenes and pyroxene-rich mineral assemblages (e.g. some meteorites - see below). Spectra of some olivines show similar features, although the most significant absorption is centered on $0.45 \mu \mathrm{m}$. Optical spectra of Ti-bearing clinopyroxenes often exhibit such complex depression, for example, as in the absorption spectra of a titanaugite and Allende (CV3) fassaite studied by Burns et al. (1976), and reflectance spectra of Ti-bearing clinopyroxenes presented by Cloutis (2002). In general, pyroxenes show extreme spectral diversity, sometimes lack two typical near infrared bands at 0.9 and $2 \mu \mathrm{m}$, and may exhibit a variety of different absorption features in the visible due to the presence of trace elements. The work of Cloutis (2002) provided some examples of this extreme spectral diversity. Pyroxenes are not the only possible candidates causing the broad inflection between 0.45 and $0.55 \mu \mathrm{m}$. Cloutis \& Burbine (1999) reported that common Fe-sulfides (troilite, pyrrhotite and intermediate phases) may be spectrally diverse and show significant spectral variations in the visible and near-infrared as a function of composition, as well as heating and oxidation degree. Some of the Fe-sulfide spectra show similar inflections.

The feature at $0.52 \mu \mathrm{m}$ might be due to traces of $\mathrm{Fe}^{3+}$ in the tetrahedral sites of clinopyroxenes. Even if only traces of $\mathrm{Fe}^{3+}$ are present in tetrahedral sites, absorptions of tetrahedral $\mathrm{Fe}^{3+}$ are predicted to be amplified with respect to those of octahedral $\mathrm{Fe}^{3+}$. Spin-forbidden transitions in tetrahedral $\mathrm{Fe}^{3+}$ (i.e., $\mathrm{Fe}^{3+}$ substitutes for $\mathrm{Si}$ in $\mathrm{SiO}_{4}$ tetrahedral) produce absorption at $\sim 0.52-0.53 \mu \mathrm{m}\left({ }^{6} \mathrm{~A}_{1} \rightarrow{ }^{4} \mathrm{~T}_{1}(\mathrm{G})\right)$. Another corresponding transition produces bands at $\sim 0.48-0.49 \mu \mathrm{m}\left({ }^{6} \mathrm{~A}_{1} \rightarrow{ }^{4} \mathrm{~T}_{2}(\mathrm{G})\right)$, and $\sim 0.42-0.44 \mu \mathrm{m}\left({ }^{6} \mathrm{~A}_{1} \rightarrow{ }^{4} \mathrm{~A}_{1}{ }^{4} \mathrm{E}(\mathrm{G})\right)$, although the exact positions may vary from mineral to mineral (Faye \& Hogarth 1969; Cohen 1972; Burns et al. 1976). The latter bands may also be present in the spectrum of Lutetia contributing to the broad depression around $0.5 \mu \mathrm{m}$. In minerals containing tetrahedral $\mathrm{Fe}^{3+}$, the $0.42-0.44 \mu \mathrm{m}$ band is usually the most intense, while other bands are broader and weaker if spectra are measured at room temperature. At lower temperatures, absorption features become narrower (sharper) and relative band intensities may change (e.g. Bell et al. 1975), which may explain why the $0.52 \mu \mathrm{m}$ band becomes resolvable in the spectrum of Lutetia. We note that some shifts of band positions in the visible range as a function of temperature are also possible (Bell et al. 1975), although it is difficult to predict the exact behavior. Another possible contribution to this band at its short-wavelength side is a well-known $0.505-0.510 \mu \mathrm{m}$ band typical of pyroxenes, being due to spin-forbidden crystal field transitions in $\mathrm{Fe}^{2+}$ (e.g. Hazen et al. 1978). It moves to longer wavelengths with increasing $\mathrm{Fe}^{2+}$ and $\mathrm{Ca}^{2+}$ content in pyroxenes (Hazen et al. 1978).

The $0.47 \mu \mathrm{m}$ band may be due to spin-allowed crystal field transitions in $\mathrm{Ti}^{3+}$ in pyroxene M1 sites (usually at 0.455-0.475 $\mu \mathrm{m}$ ), although Hazen et al. (1978) notes that $0.475 \mu \mathrm{m}$ band is also prominent in the spectra of Fe-rich orthopyroxenes, where it is due to $\mathrm{Fe}^{2+}$. Hazen et al. (1978) suggested that in lunar pyroxenes the band is due to the superposition of $\mathrm{Ti}^{3+}$ and $\mathrm{Fe}^{2+}$ effects. Cloutis (2002) noted the band at $0.46 \mu \mathrm{m}$ in his reflectance spectra of Ti-bearing high-Ca pyroxenes and suggested that it was due to $\mathrm{Fe}^{2+} \rightarrow \mathrm{Ti}^{4+}$ charge transfer. Cloutis \& Hudon (2004) also reported that the band at
$0.46 \mu \mathrm{m}$ is present in reflectance spectra of Fe-bearing spinels, where its position correlates with $\mathrm{Fe}^{2+}$ content. In addition, Cloutis (2001) detected the $0.46 \mu \mathrm{m}$ feature in reflectance spectra of millilites (components of CAIs in carbonaceous chondrites) and tentatively assigned this to spin-allowed transitions in $\mathrm{Fe}^{2+}$.

We note that although ferric iron in phyllosilicates may contribute to both the $0.43 \mu \mathrm{m}$ and $0.8 \mu \mathrm{m}$ bands (due possibly to $\mathrm{Fe}^{2+} \rightarrow \mathrm{Fe}^{3+}$ charge transfer) seen in our previous Lutetia spectra (Lazzarin et al. 2004b), the same features may also be caused by traces of ferric iron in clinopyroxene(s), or traces of ferric iron in clinopyroxene can at least contribute to these absorptions. The $0.43 \mu \mathrm{m}$ band due to spin-forbidden transitions in $\mathrm{Fe}^{2+}$ is common in visible spectra of Fe-bearing orthopyroxenes (Cloutis 2002; Klima et al. 2007, and references therein).

The broad feature around $0.45-0.55 \mu \mathrm{m}$ is present in reflectance spectra of some meteorites including some ordinary and carbonaceous chondrites, HED meteorites (especially diogenites), and ureilites. However, ordinary chondrites and HED achondrites exhibit deep absorption bands of mafic silicates in the near-infrared, while Lutetia is relatively featureless in that spectral range (Howell et al. 1994; Burbine \& Binzel 2002; Birlan et al. 2004, 2006; Nedelcu et al. 2007). Among meteorites with relatively featureless NIR spectra, a broad inflection mentioned above is present in the spectra of some chondrites of CR clan (including $\mathrm{CH}$ ) and ureilites. Nearly all spectra of ureilites (Cloutis \& Hudon 2004; Gaffey 1976) exhibit such a feature. Reflectance spectra of some ureilites also show a weak feature at $0.52 \mu \mathrm{m}$ superimposed on a broader depression between 0.45 and $0.55 \mu \mathrm{m}$. Ureilites are carbon-bearing achondrites containing olivine and several types of pyroxene i.e. pigeonite, augite, and sometimes low-Ca pyroxene. Some CO3 and CV3 chondrites, which are favored by several authors as spectral analogues of Lutetia (Birlan et al. 2004; Barucci et al. 2005, 2008), also show a broad inflection around $0.5 \mu \mathrm{m}$, but it is weaker than the features in the spectra of meteorites mentioned above. We note that in the visible spectrum of Lutetia from Barucci et al. (2005) there appears to be an absorption band at $0.5 \mu \mathrm{m}$.

By now, Lutetia has been observed for many spectral ranges, and many surface compositions have been suggested for this object. In the following, we analyze all the available data, including our own observations, in attempting to identify which composition(s) agree with observational data in a wide spectral range.

Barucci et al. (2008) published and analyzed a Spitzer Space Telescope (SST) emission spectrum of Lutetia between 7.5 and $38 \mu \mathrm{m}$ and concluded that only fine-grained CV3 and CO3 chondrites reproduced the Lutetia spectrum in this spectral range. Our own comparison of the published SST spectrum of Lutetia with available spectra of various minerals and meteorites, including heated and laser irradiated powders, confirms Lutetia's affinity with fine-grained $\mathrm{CO} 3$ and some $\mathrm{CV} 3$ meteorites, and shows that the majority of meteorite classes provide only a poor match to the thermal infrared (TIR) spectrum. For example, TIR spectra of E-chondrites, CI chondrites, and Kaidun meteorite, suggested by Nedelcu et al. (2007) as possible Lutetia' analogues based on their visible and near-infrared (VNIR) spectra, do not match the SST spectrum of Lutetia (Fig. 3). The latter spectrum shows a well-developed transparency feature centered between 12 and $13 \mu \mathrm{m}$, a smooth plateau between 9.5 and $11.5 \mu \mathrm{m}$, and an emissivity maximum (Christiansen feature) between 9.2 and $9.5 \mu \mathrm{m}$ (Fig. 3), indicating that the silicate fraction of a asteroid surface in the TIR range is spectrally dominated by fine-grained olivine (possibly Fe-rich). Among known meteorites with measured TIR spectra, only some carbonaceous meteorites show 


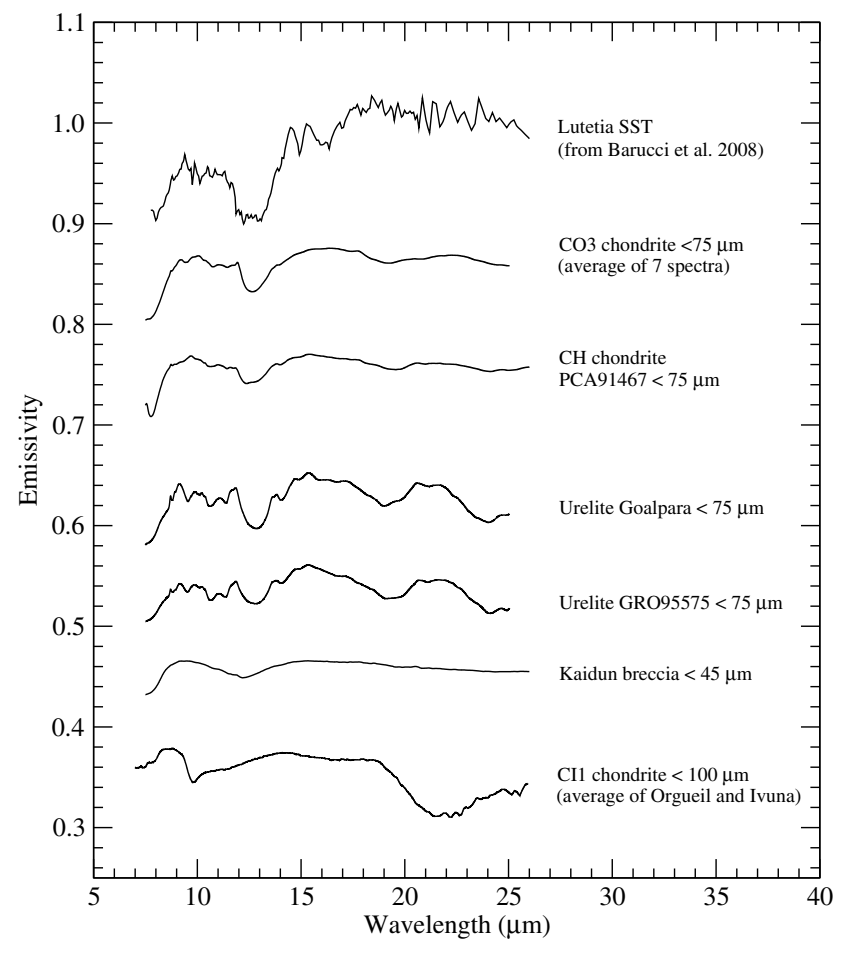

Fig. 3. The average emissivity spectrum of Lutetia from Barucci et al. (2008) compared to several spectra of carbonaceous chondrites and achondrites acquired at the NASA RELAB facility. Particle sizes in microns are indicated on the plots. The biconical reflectance $(R)$ spectra of meteorite separates were converted to emissivity spectra $(E)$ according to the Kirchhoff's law $(E=1-R)$. For clarity, each spectrum is offset by 0.1 from the previous one.

similar TIR spectral properties. We found that along with CV3 and some CO3 chondrites, mentioned by Barucci et al. (2008), the TIR spectra of two ureilites (Goalpara and GRO95575) and a CH-chondrite PCA91467 from the RELAB Spectral Database ${ }^{1}$ are consistent with the Lutetia SST spectrum (Fig. 3). The latter meteorites show relevant depressions between 0.45 and $0.55 \mu \mathrm{m}$ in their visible spectra.

However it should be noted that Rivkin et al. (2000) and Birlan et al. (2006) reported a $3 \mu \mathrm{m}$ feature of hydration in the NIR spectra of Lutetia. If this feature is real, then anhydrous carbonaceous meteorites, such as ureilites, $\mathrm{CO} 3$, and $\mathrm{CV} 3$ chondrites are poor analogues for Lutetia surface composition. Although some $\mathrm{CO} 3$ and $\mathrm{CV} 3$ meteorites show signatures of aqueous alteration on a microscopic scale (Tomeoka \& Buseck 1982, 1990; Keller et al. 1994), on a macroscopic scale they can be considered as anhydrous, since their reflectance spectra lack hydration bands at 2.7-3 $\mu \mathrm{m}$ (Larson et al. 1979; Jones 1988). The $2.7 \mu \mathrm{m}$ bands due to O-H stretch in hydrated silicates are absent in laboratory reflectance spectra of $\mathrm{CO} 3$ and $\mathrm{CV} 3$ chondrites, while weak bands at $3 \mu \mathrm{m}$ sometimes detectable in their spectra are due to adsorbed water (Jones 1988). In contrast, the metal-rich carbonaceous $\mathrm{CH}$ and $\mathrm{CB}$ chondrites contain lumps of extensively hydrated matrix material (Greshake et al. 2002), which can produce detectable $3 \mu \mathrm{m}$ bands in reflectance spectra of these meteorites. In addition, a high content of coarse metal can increase geometric albedo to a high value typical of Lutetia. Unfortunately, no spectra of CB-chondrites have yet been published, but the only available spectrum of a fine $(<75 \mu \mathrm{m})$ separated $\mathrm{CH}$ chondrite PCA91467 from the RELAB

${ }^{1}$ http://lf314-rlds.geo.brown.edu
Spectral Database shows features consistent with Lutetia spectra - nearly featureless NIR spectrum (except for the 2.7-3 $\mu \mathrm{m}$ range, and a weak band near $0.9 \mu \mathrm{m}$ ), a depression between 0.45 and $0.55 \mu \mathrm{m}$, and the TIR spectrum resembling that of Lutetia (Fig. 3).

We note that all published visible spectra of Lutetia (Bus \& Binzel 2002; Lazzarin et al. 2004b; Barucci et al. 2005), including ours, have relatively flat UV-Vis spectral slopes compared to available spectra of meteorite analogues mentioned above, especially at wavelengths shorter than $0.5 \mu \mathrm{m}$. All meteorites mentioned above contain ferrous and/or ferric iron, and therefore their spectra show metal-oxygen charge-transfer absorption towards the UV. We can suggest several possible explanations for the flatter UV-Vis slopes in the spectra of Lutetia. Its regolith may be enriched in opaque components (metal and/or sulfides), either originally, or due to surface alteration processes such as shock or space weathering. However, the high albedo of Lutetia makes the space weathering option unlikely. Although space weathering simulations tend to flatten UV-Vis slopes (e.g. Lazzarin et al. 2006; Noble et al. 2007), severe alteration accompanied by significant decreases in albedo would probably be needed to produce a slope typical of Lutetia. High contents of coarse-grained opaques would not significantly affect the shape of a TIR spectrum and the positions of its features. Another possibility could be the extremely fine grain size of silicate components, which can decrease the intensity of the UVabsorption band, and hence, the UV-Vis slope (e.g. Mustard \& Hays 1997). This possibility agrees well with the SST spectrum of Barucci et al. (2008), indicating that silicates on Lutetia's surface are very fine-grained. Similar flattening of the UV-Vis slope could also exist in case of a large silicate grain size, causing the UV-band saturation, but this would be inconsistent with the shape of the SST spectrum from Barucci et al. (2008). Finally, available meteorite assemblages may not be representative analogues of the Lutetia surface composition, and its flat UV-visual slope could be explained by the low Fe-content in silicates exposed at its surface. Some Fe in silicates should be present, however, in order to explain the presence of weak features observed in the visible spectra of Lutetia.

\section{Conclusions}

We have discussed the presence of absorption bands in visible spectra of (21) Lutetia, a target of the Rosetta mission. We obtained two new spectra of this asteroid with different spectral resolutions. We detected a broad inflection between 0.45 and $0.55 \mu \mathrm{m}$ and two minor bands around 0.47 and $0.52 \mu \mathrm{m}$, partially confirming our previous results. We suggest that these features could be caused by charge transfer involving various metal ions in pyroxenes. At this stage, summarizing available results, we conclude that Lutetia is a rather unusual asteroid. The spectral range of the new visible spectra presented here $(0.45-0.7 \mu \mathrm{m})$ is too narrow for reliable taxonomic classification, but our previous spectra (Lazzarin et al. 2004b) are consistent with C-class of Bus taxonomy (Bus 1999). Although the radar albedo was reported to be low, IR observations and thermal modeling (Mueller et al. 2006) showed that the albedo is high and consistent with the IRAS albedo. The geometric albedo of Lutetia is too high for it to be a C-class object, but it is not inconsistent with the possible presence in this asteroid of some carbonaceous meteorites. Most laboratory spectra of meteorites have been acquired at non-zero phase angles, while photometric measurements performed as a function of phase angle show that the reflectance of carbonaceous meteorites increases 
drastically at small phase angles (Kamei \& Nakamura 2002; Sakai \& Nakamura 2005), especially if the material is finegrained and porous (Okada et al. 2006). For example, normal reflectance of a fine-grained $(<45 \mu \mathrm{m}) \mathrm{CV} 3$ chondrite Allende is as high as 0.22 at $0.6 \mu \mathrm{m}$ (Kawakami \& Nakamura 2007). It remains unclear why C-class asteroids are generally far darker then carbonaceous chondrite powders. Darkening due to impacts and space weathering could explain the difference. In this case, "bright" Lutetia might represent a body with a relatively "fresh" surface. Although the large mean collisional age of this object suggests a high exposure value and thus a high degree of space weathering (Lazzarin et al. 2006; Paolicchi et al. 2007), its surface may have been refreshed considerably by non-catastrophic collisions. This could be consistent with the work of Carvano et al. (2008), who suggested that some discrepancies between albedo and thermal inertia values for Lutetia derived from various TIR datasets can be explained by the presence of large craters in its northern hemisphere. Alternative explanation for the high albedo of Lutetia compared to C-class objects is that Lutetia's surface may be enriched in bright (coarse-grained), opaque phases, similarly to metal-rich carbonaceous chondrites, or its surface could be enriched in fine-grained bright silicates. Finally, it is possible that Lutetia belongs to the X-class instead of C-class, as suggested by several researchers, but this in no way precludes a mineral assemblage related to carbonaceous meteorites. Our analysis of Lutetia spectra in a wide spectral range, covered with different observations, suggests that metal-rich carbonaceous chondrites currently seem to be the most plausible analogue materials. However, more observations of Lutetia and more laboratory measurements of meteorites are needed to draw reliable conclusions regarding the surface composition of the asteroid.

Acknowledgements. We thank S. Mottola for helpful discussions. We thank M.A. Barucci who provided us with the average SST spectrum of Lutetia (Barucci et al. 2008). This research utilizes spectra acquired by T. Hiroi, D.W. Mittlefeldt and P. Hudon with the NASA RELAB facility at Brown University. The work of L.V. Moroz is supported by DLR MERTIS project 50 QW 0502.

\section{References}

Barucci, M. A., Capria, M. T., Coradini, A., \& Fulchignoni, M. 1987, Icarus, 72, 304

Barucci, M. A., Fulchignoni, M., Fornasier, S., et al. 2005, A\&A, 430, 313

Barucci, M. A., Fornasier, S., Dotto, E., et al. 2008, A\&A, 477, 665

Bell, III, J. F., Lucey, P. G., Owensby, P. D., \& McCord, T. B. 1989, in BAAS, 21,954

Bell, P. M., Mao, H. K., \& Rossman, G. R. 1975, Absorption spectroscopy of ionic and molecular units in crystals and glasses, Infrared and Raman Spectroscopy of Lunar and Terrestrial Minerals, ed. C. J. Karr (New York: Academic Press), 1

Belskaya, I. N., \& Lagerkvist, C.-I. 1996, Planet. Space Sci., 44, 783

Birlan, M., Barucci, M. A., Vernazza, P., et al. 2004, New Astronomy, 9, 343

Birlan, M., Vernazza, P., Fulchignoni, M., et al. 2006, A\&A, 454, 677

Burbine, T. H., \& Binzel, R. P. 2002, Icarus, 159, 468

Burns, R. G., Parkin, K. M., Loeffler, B. M., Leung, I. S., \& Abu-Eid, R. M. 1976, in Lunar and Planetary Science Conference, ed. D. C. Kinsler, 7, 2561
Bus, S. J. 1999, Ph.D. Thesis, Massachusetts Institute of Technology Bus, S. J., \& Binzel, R. P. 2002, Icarus, 158, 146

Carvano, J. M., Barucci, M. A., Delbó, M., et al. 2008, A\&A, 479, 241

Cloutis, E. A. 2001, in Lunar and Planetary Institute Conference Abstracts, 32, 1128

Cloutis, E. A. 2002, J. Geophys. Res. (Planets), 107, 5039

Cloutis, E. A. \& Burbine, T. H. 1999, in Lunar and Planetary Institute Conference Abstracts, 30, 1875

Cloutis, E. A. \& Hudon, P. 2004, in Lunar and Planetary Institute Conference Abstracts, ed. S. Mackwell, \& E. Stansbery, 35, 1257

Cohen, A. J. 1972, Moon, 4, 141

Dotto, E., Barucci, M. A., Fulchignoni, M., et al. 1992, A\&AS, 95, 195

Faye, G. H., \& Hogarth, D. D. 1969, Can Mineral, 10, 25

Gaffey, M. J. 1976, J. Geophys. Res., 81, 905

Greshake, A., Krot, A. N., Meibom, A., et al. 2002, Meteoritics and Planetary Science, 37, 281

Hazen, R. M., Bell, P. M., \& Mao, H. K. 1978, in Lunar and Planetary Science Conference, 9, 2919

Hicks, M. D., Bauer, J. M., \& Tokunaga, A. T. 2004, IAU Circ., 8315, 3

Hiroi, T., Bell, J. F., Takeda, H., \& Pieters, C. M. 1993, Icarus, 102, 107

Howell, E. S., Merenyi, E., \& Lebofsky, L. A. 1994, J. Geophys. Res., 99, 10847

Jones, T. D. 1988, Ph.D. Thesis, AA(Arizona Univ., Tucson.)

Kamei, A., \& Nakamura, A. M. 2002, Icarus, 156, 551

Kawakami, K., \& Nakamura, A. M. 2007, in Lunar and Planetary Institute Conference Abstracts, 38, 1531

Keller, L. P., Thomas, K. L., Clayton, R. N., et al. 1994, Geochim. Cosmochim. Acta, 58, 5589

Klima, R. L., Pieters, C. M., \& Dyar, M. D. 2007, Meteoritics and Planetary Science, 42, 235

Lagerkvist, C.-I., Erikson, A., Debehogne, H., et al. 1995, A\&AS, 113, 115

Larson, H. P., Feierberg, M. A., Fink, U., \& Smith, H. A. 1979, Icarus, 39, 257

Lazzarin, M., Marchi, S., Barucci, M. A., di Martino, M., \& Barbieri, C. 2004a, Icarus, 169,373

Lazzarin, M., Marchi, S., Magrin, S., \& Barbieri, C. 2004b, A\&A, 425, L25

Lazzarin, M., Marchi, S., Moroz, L. V., et al. 2006, ApJ, 647, L179

Lazzaro, D., Angeli, C. A., Carvano, J. M., et al. 2004, Icarus, 172, 179

Luu, J. X., \& Jewitt, D. C. 1990, AJ, 99, 1985

Magri, C., Ostro, S. J., Rosema, K. D., et al. 1999, Icarus, 140, 379

Mueller, M., Harris, A. W., Bus, S. J., et al. 2006, A\&A, 447, 1153

Mustard, J. F., \& Hays, J. E. 1997, Icarus, 125, 145

Nedelcu, D. A., Birlan, M., Vernazza, P., et al. 2007, A\&A, 470, 1157

Noble, S. K., Pieters, C. M., \& Keller, L. P. 2007, Icarus, 192, 629

Okada, Y. A., Nakamura, M., \& T., M. 2006, J. Quant. Spec. Radiat. Transf., 100,295

Paolicchi, P., Marchi, S., Nesvorný, D., Magrin, S., \& Lazzarin, M. 2007, A\&A, 464, 1139

Prokof'eva, V. V., Bochkov, V. V., \& Busarev, V. V. 2005, Sol. Sys. Res., 39, 410

Rivkin, A. S., Howell, E. S., Lebofsky, L. A., Clark, B. E., \& Britt, D. T. 2000, Icarus, 145,351

Sakai, T., \& Nakamura, A. M. 2005, Earth, Planets, and Space, 57, 71

Shepard, M. K., Clark, B. E., Benner, L. A. M., et al. 2005, in BAAS, 37, 628

Soderblom, L. A. 1992, The composition and mineralogy of the Martian surface from spectroscopic observations -0.3 micron to 50 microns (Mars), 557

Tedesco, E. F., Veeder, G. J., Fowler, J. W., \& Chillemi, J. R. 1992, The IRAS Minor Planet Survey, Tech. rep.

Tholen, D. J., \& Barucci, M. A. 1989, in Asteroids II, ed. R. P. Binzel, T. Gehrels, \& M. S. Matthews, 298

Tomeoka, K., \& Buseck, P. R. 1982, Nature, 299, 326

Tomeoka, K., \& Buseck, P. R. 1990, Geochim. Cosmochim. Acta, 54, 1745

Torppa, J., Kaasalainen, M., Michalowski, T., et al. 2003, Icarus, 164, 346

Vilas, F., Hatch, E. C., Larson, S. M., Sawyer, S. R., \& Gaffey, M. J. 1993, Icarus, 102,225

Zappala, V., di Martino, M., Knezevic, Z., \& Djurasevic, G. 1984, A\&A, 130, 208

Zellner, B., Tholen, D. J., \& Tedesco, E. F. 1985, Icarus, 61, 355 